\title{
BMJ Open Effectiveness of non-pharmacological interventions for reducing postpartum fatigue: a systematic review protocol
}

\author{
Jialu Qian, ${ }^{1,2}$ Shiwen Sun, ${ }^{2}$ Lu Liu, ${ }^{1,2}$ Xiaoyan Yu (D) ${ }^{2}$
}

To cite: Qian J, Sun S, Liu L, et al. Effectiveness of nonpharmacological interventions for reducing postpartum fatigue: a systematic review protocol. BMJ Open 2021;11:e051136. doi:10.1136/ bmjopen-2021-051136

- Prepublication history and additional supplemental material for this paper are available online. To view these files, please visit the journal online (http://dx.doi.org/10.1136/ bmjopen-2021-051136).

Received 10 March 2021 Accepted 08 0ctober 2021

Check for updates

(C) Author(s) (or their employer(s)) 2021. Re-use permitted under CC BY-NC. No commercial re-use. See rights and permissions. Published by BMJ.

${ }^{1}$ School of Nursing, Zhejiang University School of Medicine, Hangzhou, Zhejiang, China ${ }^{2}$ Department of Obstetrics, Zhejiang University School of Medicine Women's Hospital, Hangzhou, Zhejiang, China

Correspondence to Professor Xiaoyan Yu; yuxy@zju.edu.cn

\section{ABSTRACT}

Introduction Postpartum fatigue is a common symptom among new mothers after their pregnancy. It has a considerable negative impact on women's functional and mental status as well as the development of babies. Identifying effective interventions to prevent or reduce postpartum fatigue is meaningful to improve the quality of life and avoid adverse outcomes of this vulnerable population. This systematic review aims to synthesise nonpharmacological evidence and evaluate the effectiveness of interventions for reducing postpartum fatigue among puerperas.

Methods and analysis This review will be conducted in accordance with the Preferred Reporting Items for Systematic Review and Meta-Analysis Protocols. We will systematically search the Cochrane Library, PubMed, Embase, Web of Science, PsycINFO, CINAHL and ProQuest databases to identify clinical trials implementing nonpharmacological interventions conducted during 0-78 weeks postpartum for fatigue reduction. An additional search of OpenGrey will be conducted to identify grey literature. The search will be performed on 30 March 2021 without restrictions on time and language. Two independent reviewers will be responsible for study selection, data extraction and study quality assessment. The Cochrane risk-of-bias tool will be adopted to evaluate the risk biases of the included randomised controlled trials, and the Risk of Bias in Non-randomised Studies of Interventions will be applied to evaluate non-randomised controlled trials. Any disagreements will be referred to a third reviewer to reach a consensus. Findings will be qualitatively synthesised, and a meta-analysis will be conducted for the statistical combination if outcome data are sufficient and available.

Ethics and dissemination This systematic review will not involve the collection of primary data and will be based on published data. Therefore, ethics approval is not required. The final findings will be disseminated through peerreviewed journals and academic conferences. PROSPERO registration number CRD42021234869

\section{INTRODUCTION}

Postpartum fatigue is defined as tiredness and decrease in physical and mental capacity. ${ }^{1}$ Approximately $64 \%$ of new mothers are affected by fatigue in the postpartum stage, making it the most common and persistent issue that women face when they adapt to

\section{Strengths and limitations of this study}

This will be the first systematic review to summarise the non-pharmacological interventions to reduce postpartum fatigue among puerperae.

- The protocol adheres to the Preferred Reporting Items for Systematic Review and Meta-Analysis Protocols guidelines.

- Rigorous methods of review will be followed with at least two independent reviewers to perform study selection, data extraction and critical appraisal.

- The databases will be searched without time and language restrictions.

- A potential limitation may be that non-English electronic databases will not be searched, which may cause language bias.

motherhood. ${ }^{2}{ }^{3}$ Substantial proportions of women, $38.8 \%, 27.1 \%$ and $11.4 \%$ perceived fatigue at 10 days, 1 month and 3 months after birth, respectively. ${ }^{4}$ The severity of postpartum fatigue can be affected by sociodemographic (eg, education level or age), physiological (eg, birth modes, feeding patterns, durations of the second stage of labour or perineal pain) and psychosocial (eg, depression, stress, anxiety or social support) factors. ${ }^{24-8}$

Postpartum fatigue is an unrelenting condition that can cause an overwhelming feeling of exhaustion in the mother, bringing about a decrease in their physical and mental ability to work and seriously affecting maternal health and babies. ${ }^{9}$ It is a long-term postpartum health problem and has persistent adverse influences on mothers' physical and mental health. ${ }^{10}$ Postpartum fatigue is negatively associated with sleep quality. ${ }^{11} 12$ It may increase the risk of depression, anxiety and sleep problems. ${ }^{461314}$

In terms of adverse outcomes of postpartum fatigue for their offspring, it is frequently related to inadequate breastmilk production and babies' weaning off breastmilk earlier. ${ }^{15-17}$ Postpartum fatigue is recognised as one of the factors that hinder the initiation of lactation. ${ }^{18}$ Experiences of fatigue after 
birth may lead to less effective parenting behaviours and negative influences on maternal-infant attachment. ${ }^{19}$ Previous research found a negative correlation between postpartum fatigue and parenting sense of competence. ${ }^{20}$ Incremental exhaustion diminished parents' ability to concentrate, triggering distress symptoms and burdening parent-child interaction, ${ }^{21}$ even delaying the development of babies. ${ }^{22}$

Strategies for postpartum fatigue reduction are not only important to women's physical relief but also less stigmatising initial prevention strategies for psychological issues compared with recommended psychotherapy. ${ }^{23}$ However, postpartum fatigue is minimally examined and there is limited research for the effective treatment of it. ${ }^{23}$ Hence, it is critical to deliberately prevent and decrease fatigue during the postpartum period through effective approaches. Mothers have to breastfeed their babies in postpartum period. Comparing with pharmacological interventions, non-pharmacological interventions are safer for both mothers and babies. Non-pharmacological interventions are also more convenient to be applied in practice. Therefore, this review will focus on nonpharmacological interventions for reducing postpartum fatigue.

To date, a number of studies have attempted to manage and reduce postpartum fatigue via non-pharmacological interventions. In recent trials, various forms of postpartum exercise $^{24-33}$ including yoga, aerobic gymnastic exercise and Pilates exercises have been examined to explore effects on reducing fatigue in puerperae. However, their effectiveness varied. Psychoeducational programmes delivered via home visits, booklets and home calls were implemented to manage postpartum fatigue, but consistent conclusion was not drawn. ${ }^{3435}$ Given that labour pain is an influencing factor of postpartum fatigue, some studies tried to decrease postpartum fatigue from the perspective of managing labour pain ${ }^{3637}$ and positive effects were found. In addition, physical therapies consisting motherinfant skin-to-skin contact, ${ }^{38}$ taking warm showers ${ }^{39}$ and breathing lavender oil aroma ${ }^{40}$ were used for postpartum fatigue reduction. Moreover, other non-pharmacological interventions, including drinking tea ${ }^{4-43}$ (drink one cup of chamomile tea every day) and laughter therapy ${ }^{44}$ showed benefits to reduce fatigue symptoms among puerperas. As can be seen, although studies of postpartum fatigue management interventions conducted in mothers are accumulating, consistent conclusion is not obtained for a certain intervention and different interventions may lead to diverse effects on postpartum fatigue.

Relevant systematic reviews have analysed the relationship of postpartum fatigue and depression, ${ }^{45}$ explored the effects of exercise on pregnancy and postpartum fatigue $^{46}$ and identified predictive factors of postpartum fatigue. ${ }^{47}$ To the best of our knowledge, there is currently no in-depth systematic review on non-pharmacological interventions specific for postpartum fatigue. Therefore, this protocol illustrates the rationale and details of the planned systematic review and meta-analysis. Considering that postpartum fatigue duration is in direct proportion to the severity of related health effects, it ranges from the first postpartum weeks to several months. ${ }^{48}$ A previous systematic review ${ }^{45}$ indicated that the longest follow-up time of postpartum fatigue is 78 weeks after delivery. Therefore, we define postpartum as $0-78$ weeks after giving birth in our study.

\section{Objectives}

This study aims to synthesise the scientific literature and, if possible, perform a meta-analysis to clarify the effectiveness of diverse non-pharmacological interventions for reducing postpartum fatigue in puerperae. We would like to address the following research question: What nonpharmacological interventions decreased postpartum fatigue (or not), and why were they effective (or not)?

\section{METHODS AND ANALYSIS}

\section{Study design}

The systematic review protocol adheres to the Preferred Reporting Items for Systematic Review and Meta-Analysis Protocols (PRISMA-P) guidelines. ${ }^{49}$ The PRISMA-P checklist can be seen in online supplemental file 1 . The PRISMA guidelines will also be used for this systematic review to guide and ensure transparent and rigorous reporting. ${ }^{50}$ We developed the research questions based on the population, intervention, comparison or control, and outcome framework, which are described in detail below.

\section{Eligibility criteria for selecting studies \\ Types of studies}

This systematic review will include randomised or nonrandomised controlled trials (RCTs). Clinical trials adopting RCTs, quasi-RCTs, before-and-after or prospective cohort study designs. Only studies published in English will be included.

\section{Participants}

The participants will be puerperas aged 18 years or over who have had a healthy delivery regardless of their gravidity and parity.

\section{Interventions}

Any type of non-pharmacological intervention conducted during 0-78 weeks postpartum with either the primary or secondary purpose of decreasing fatigue will be eligible. The setting, frequency, timing and duration of the interventions will not be limited.

The potential interventions may include but are not limited to the following:

- Exercise.

- Psychoeducation programme.

- Labour pain management interventions.

- Mother-infant skin-to-skin contact.

- Taking warm showers.

- Aromatherapy.

- Refloxology. 
- Drinking tea.

- Laughter therapy.

\section{Controls}

Trials will be included if the intervention group is compared with controls, which may be usual care, placebo, wait-list or no interventions.

\section{Outcomes}

Postpartum fatigue estimated as the rate or mean severity of fatigue will be the primary outcome in this systematic review. Postpartum fatigue could be evaluated using the following valid measurements but not restricted to:

- The Postpartum Fatigue Scale.

- The Lee Fatigue Scale.

- The Fatigue Identification Form.

- The Fatigue Severity Scale.

If possible, we will examine psychological variables (such as depression and stress).

\section{Exclusion criteria}

The following studies will be excluded: (1) duplicated publications (only the one with the most participants will be included), (2) analyses of interventions based on postpartum fatigue in some special conditions, such as postpartum haemorrhage and (3) studies without sufficient data to be extracted.

\section{Search strategies}

Systematic literature searches will be conducted from inception to 31 March 2021. We will comprehensively search the following electronic databases without limitations on time and language: Cochrane Library, PubMed, Embase, Web of Science, PsycINFO, CINAHL and ProQuest. An additional search of OpenGrey will be conducted for grey literature. The reference lists of the included articles and reviews related to postpartum fatigue will be carefully reviewed to retrieve eligible studies. The first author and an academic librarian will be responsible for the development of search strategies. We will use a combination of Medical Subject Heading/Emtree terms and free terms. The detailed search strategy for PubMed is displayed in online supplemental file 2. Two reviewers will perform the search process independently.

\section{Selection of studies}

The identified references will be imported into EndNote V. X9 software for better data management. After removing duplicates, reviews and protocols, two reviewers will independently screen the studies according to the inclusion criteria in two stages: (1) title and abstract screening and (2) full-text screening. Each potential article will be labelled 'include', 'exclude' or 'unclear'. The full texts of studies labelled 'include' or 'unclear' will be reviewed, and two reviewers will then carefully determine whether to include these studies. If any discrepancy appeared, a third author was consulted to reach a consensus decision after discussion. A PRISMA flow will be used to provide detailed information on the study selection process. ${ }^{51}$

\section{Data extraction}

Two reviewers will adopt a standardised data extraction table to independently extract and record data from the included studies. Medical Research Council guidance and relevant previous studies that have clarified vital components for process evaluations ${ }^{52-54}$ will guide the data extraction table. The data extraction table will be piloted with a subsample of included articles and then further discussed and revised by our research members. Study authors will be contacted to request missing data. Inconsistencies between two reviewers will be discussed and resolved by referring to a third reviewer.

The following information was extracted from the included studies:

1. Publication information: first author, country and publication year.

2. Participant: Participant characteristics (age, gravidity, parity, type of delivery, etc.) and sample size (intervention/control).

3. Intervention details: type, content, frequency, duration and control group.

4. Outcomes: fatigue rate or severity, psychological variables (such as depression and stress), evaluation time points and assessment tools.

\section{Risk of bias}

For the included RCTs, we will use the Cochrane risk-ofbias tool for quality assessment. ${ }^{55}$ The following seven areas will be evaluated: random sequence generation, allocation concealment, blinding of participants and researchers, blinding of outcome assessors, incomplete outcome data, selective reporting and other bias. Bias was evaluated as a judgement (high or low or unclear), and then each study was rated as having a high, moderate or low risk of bias according to the seven domains. The Risk of Bias in Non-randomised Studies of Interventions will be used to assess the risk of bias of non-RCTs.$^{56}$ Seven domains are included in this tool: confounding, selection of participants into the study, classification of intervention, deviation from intended interventions, missing data, measurement of outcomes and selection of the reported results. Two reviewers will independently assess the risk of bias, and disagreements will be discussed with a third researcher to reach a consistent conclusion.

\section{Data analysis}

A narrative approach will be undertaken for data synthesis in this systematic review. After comparing similarities and differences among study results and data extracted, the synthesis will give a comprehensive and detailed description. This will enhance our understanding of how postpartum fatigue was measured, the existing intervention types, especially the specific intervention contents and the effectiveness of these interventions.

We will perform a meta-analysis utilising Reviewer Manager 5.3 if possible to statistically combine no less than two studies conducting similar interventions and using the same assessment tool. For continuous outcomes, we 
will calculate mean differences and 95\% CIs. For dichotomous data, risk ratios and $95 \%$ CIs will be computed.

The heterogeneity between the analysed trials will be examined by $\chi^{2}$ test and $I^{2}$ test. If the $\mathrm{p}$ value is $>0.1$ or $I^{2}<50 \%$, it indicates that there is no observed heterogeneity, and the researchers will employ a fixed effects model to combine the study results. Otherwise, a random effects model will be used. ${ }^{57}$

If there are sufficient studies, we will be able to perform subgroup analyses for primary outcome based on countries, characteristics of participants, design of interventions (such as frequency, duration or length of follow-up assessment) and outcome measurements.

In terms of the assessment of publication bias, we plan to conduct Egger's test ${ }^{58}$ and funnel plots when analysing more than 10 trials. In addition, sensitivity analysis for the primary outcome will be performed to investigate the robustness of the findings and to explore the influence of trials having high risk bias. Then, studies with a lower quality should be excluded regarding their sample size, evidence of their strength and the impact of the size grouped effect.

\section{Grading quality of evidence}

The Grading of Recommendation Assessment, Development and Evaluation system ${ }^{5960}$ will guide the evaluation of the quality of the evidence. After assessments of the risk of bias, consistency, directness of evidence, imprecision and publication bias, the quality of the evidence will be categorised as high, moderate, low and very low quality. Two reviewers will be responsible for performing quality evaluation independently.

\section{Patient and public involvement}

Since this study will be based on previously published research, patients and the public will not be directly involved in this systematic review.

\section{Ethics and dissemination}

This systematic review will be based on published data and ethics approval is not required. Following the guidelines of PRISMA-P, this protocol was registered in the International Prospective Register of Systematic Reviews (PROSPERO) on 5 March 2021. The final findings will be disseminated through publications in peer-reviewed journals, presentations at academic conferences and the doctoral thesis of the leading author.

Contributors This systematic review protocol was conceived and drafted by JLO under the supervision of XYY; JLQ and SWS will independently search electronic databases and screen titles and abstracts. JLQ and LL will be responsible for reviewing the included studies. All authors contributed to the protocol design and ongoing plan.

Funding This work was supported by Zhejiang Medical and Health Research Project (grant number2020KY173).

Competing interests None declared.

Patient consent for publication Not applicable.

Provenance and peer review Not commissioned; externally peer reviewed.

Data availability statement Data are available in a public, open access repository.
Supplemental material This content has been supplied by the author(s). It has not been vetted by BMJ Publishing Group Limited (BMJ) and may not have been peer-reviewed. Any opinions or recommendations discussed are solely those of the author(s) and are not endorsed by BMJ. BMJ disclaims all liability and responsibility arising from any reliance placed on the content. Where the content includes any translated material, BMJ does not warrant the accuracy and reliability of the translations (including but not limited to local regulations, clinical guidelines, terminology, drug names and drug dosages), and is not responsible for any error and/or omissions arising from translation and adaptation or otherwise.

Open access This is an open access article distributed in accordance with the Creative Commons Attribution Non Commercial (CC BY-NC 4.0) license, which permits others to distribute, remix, adapt, build upon this work non-commercially, and license their derivative works on different terms, provided the original work is properly cited, appropriate credit is given, any changes made indicated, and the use is non-commercial. See: http://creativecommons.org/licenses/by-nc/4.0/.

ORCID iD

Xiaoyan Yu http://orcid.org/0000-0003-3861-7340

\section{REFERENCES}

1 Doering J, Durfor SL. The process of "persevering toward normalcy" after childbirth. MCN Am J Matern Child Nurs 2011;36:258-65.

2 Badr HA, Zauszniewski JA. Meta-nalysis of the predictive factors of postpartum fatigue. Appl Nurs Res 2017;36:122-7.

3 Song J-E, Chae HJ, Kim CH. Changes in perceived health status, physical symptoms, and sleep satisfaction of postpartum women over time. Nurs Health Sci 2014;16:335-42.

4 Henderson J, Alderdice F, Redshaw M. Factors associated with maternal postpartum fatigue: an observationalstudy. BMJ Open 2019;9:e25927.

$5 \mathrm{Kim}$ J-I, Lee K-J, Symptoms B. Bladder symptoms, fatigue and physical activity in postpartum women. Asian Nurs Res 2017;11:50-5.

6 Taylor J, Johnson M. The role of anxiety and other factors in predicting postnatal fatigue: from birth to 6 months. Midwifery 2013;29:526-34.

7 Hsieh C-H, Chen C-L, Han T-J, et al. Factors influencing postpartum fatigue in Vaginal-Birth women: testing a path model. $J$ Nurs Res 2018;26:332-9.

8 Guo L, Xing W, Wang R. Status of postpartum fatigue in women of advanced maternal age and its influencing factors. Nursing Journal of Chinese People's Liberation Army 2021;38:48-51.

9 Rychnovsky JD. Postpartum fatigue in the active-duty military woman. J Obstet Gynecol Neonatal Nurs 2007;36:38-46.

10 Aksu S, Varol FG, Hotun Sahin N. Long-term postpartum health problems in Turkish women: prevalence and associations with selfrated health. Contemp Nurse 2017;53:167-81.

11 Yang Y, Li J, Zhang F. Relationship between sleep quality and postpartum fatigue. Journal of Nursing Science 2019;34:16-19.

12 Tsuchiya M, Mori E, Iwata $\mathrm{H}$, et al. Fragmented sleep and fatigue during postpartum hospitalization in older primiparous women. Nurs Health Sci 2015;17:71-6.

13 Rychnovsky J, Hunter LP. The relationship between sleep characteristics and fatigue in healthy postpartum women. Womens Health Issues 2009;19:38-44.

14 Doering JJ, Sims DA, Miller DD. How postpartum women with depressive symptoms manage sleep disruption and fatigue. Res Nurs Health 2017;40:132-42.

15 Senol DK, Yurdakul M, Ozkan SA. The effect of maternal fatigue on breastfeeding. Niger J Clin Pract 2019;22:1662-8.

16 Wang W, Lau Y, Chow A, et al. Breast-feeding intention, initiation and duration among Hong Kong Chinese women: a prospective longitudinal study. Midwifery 2014;30:678-87.

17 Brown CRL, Dodds L, Legge A, et al. Factors influencing the reasons why mothers stop breastfeeding. Can J Public Health 2014;105:e179-85.

18 Bai T, Yang Y, Fu X. Analysis of the impact of postpartum fatigue on the onset of lactation. Chin J Nursing 2018;53:438-42 https:// pesquisa.bvsalud.org/portal/resource/pt/wpr-708757

19 Lai Y-L, Hung C-H, Stocker J, Chan T-F, et al. Postpartum fatigue, baby-care activities, and maternal-infant attachment of vaginal and cesarean births following rooming-in. Applied Nursing Research 2015;28:116-20.

20 Jin L, Feng P, Xiang L. Correlation between maternal postpartum fatigue level and parenting sense of competence. Chinese Journal of Modern Nursing 2020;26:2768-72. 
21 Kurth E, Kennedy HP, Spichiger E, et al. Crying babies, Tired mothers: what do we know? a systematic review. Midwifery 2011;27:187-94

22 Lai Y-L, Hung C-H, Stocker J, et al. Postpartum fatigue, baby-care activities, and maternal-infant attachment of vaginal and cesarean births following rooming-in. Appl Nurs Res 2015;28:116-20.

23 Dennis C-L, Vigod S. Preventing postpartum depression: fatigue management is a place to start. Evid Based Nurs 2020;23:25.

24 Yang $\mathrm{C}-\mathrm{L}$, Chen $\mathrm{C}-\mathrm{H}$. Effectiveness of aerobic gymnastic exercise on stress, fatigue, and sleep quality during postpartum: a pilot randomized controlled trial. Int $J$ Nurs Stud 2018;77:1-7.

25 Dritsa M, Dupuis G, Lowensteyn I, et al. Effects of home-based exercise on fatigue in postpartum depressed women: who is more likely to benefit and why? J Psychosom Res 2009;67:159-63.

26 Ashrafinia F, Mirmohammadali M, Rajabi H, et al. Effect of Pilates exercises on postpartum maternal fatigue. Singapore Med $J$ 2015;56:169-73.

27 Lee AS, McInnes RJ, Hughes AR, et al. The effect of the more active MuMs in Stirling trial on body composition and psychological wellbeing among postnatal women. J Pregnancy 2016;2016:1-10.

28 Liu Y-H, Chang C-F, Hung H-M, et al. Outcomes of a walking exercise intervention in postpartum women with disordered sleep. $J$ Obstet Gynaecol Res 2021;47:1380-1387.

29 Gui Y. Effect of group mindfulness yoga on mothers with postpartum depression. Nursing Practice and Research 2020;17:92-3.

30 Wei X. Effect of yoga training on Postnatal/Postpartum fatigue. Chinese Evidence-Based Nursing 2020;6:734-6.

31 Ashrafinia F, Mirmohammadali M, Rajabi H, et al. Effect of Pilates exercises on postpartum maternal fatigue. Singapore Med $J$ 2015;56:169-73.

32 Yang C-L, Chen C-H. Effectiveness of aerobic gymnastic exercise on stress, fatigue, and sleep quality during postpartum: a pilot randomized controlled trial. Int J Nurs Stud 2018;77:1-7.

33 Ko Y-L, Yang C-L, Chiang L-C. Effects of postpartum exercise program on fatigue and depression during "doing-the-month" period. J Nurs Res 2008;16:177-86.

34 Giallo R, Cooklin A, Dunning M, et al. The efficacy of an intervention for the management of postpartum fatigue. J Obstet Gynecol Neonatal Nurs 2014;43:598-613.

35 Ozcan S, Eryilmaz G. Using levine's conservation model in postpartum care: a randomized controlled trial. Health Care Women Int 2021;42:794-814.

36 Xiao $\mathrm{L}$. The influence of music analgesic labor on postpartum fatigue. Journal of Mathematical Medicine 2020;33:1889-90.

37 Di Y. Effect of applying a Doula instrument of painless delivery for postpartum Fatiuge and onset time of lactation. Nursing Practice and Research 2020;17:114-6.

38 Tosun Güleroğlu F, Mucuk S, Özgürlük İzzet. The effect of motherinfant skin-to-skin contact on the involution process and maternal postpartum fatigue during the early postpartum period. Women Health 2020;60:707-18.

39 Hsieh C-H, Chen C-L, Chung F-F, et al. Efficacy of warm showers on postpartum fatigue among Vaginal-Birth Taiwanese women: a quasiexperimental design. Res Theory Nurs Pract 2017;31:96-106.

40 Vaziri F, Shiravani M, Najib FS, et al. Effect of lavender oil aroma in the early hours of postpartum period on maternal pains, fatigue, and mood: a randomized clinical trial. Int J Prev Med 2017;8:29.
41 Xue L, Zhang J, Shen $\mathrm{H}$, et al. A randomized controlled pilot study of the effectiveness of Magnolia tea on alleviating depression in postnatal women. Food Sci Nutr 2020;8:1554-61.

42 Chen S-L, Chen C-H. Effects of lavender tea on fatigue, depression, and maternal-infant attachment in Sleep-Disturbed postnatal women Worldviews Evid Based Nurs 2015;12:370-9.

43 Chang S-M, Chen $\mathrm{C}-\mathrm{H}$. Effects of an intervention with drinking chamomile tea on sleep quality and depression in sleep disturbed postnatal women: a randomized controlled trial. J Adv Nurs 2016;72:306-15.

44 Shin HS, Ryu KH, Song YA. [Effects of laughter therapy on postpartum fatigue and stress responses of postpartum women]. $J$ Korean Acad Nurs 2011;41:294-301.

45 Wilson N, Lee JJ, Bei B. Postpartum fatigue and depression: a systematic review and meta-analysis. J Affect Disord 2019;246:224-33.

46 Liu N, Wang J, Chen D-D, et al. Effects of exercise on pregnancy and postpartum fatigue: a systematic review and meta-analysis. Eur $J$ Obstet Gynecol Reprod Biol 2020;253:285-95.

47 Badr HA, Zauszniewski JA. Meta-analysis of the predictive factors of postpartum fatigue. Appl Nurs Res 2017;36:122-7.

48 Doering JJ, Dogan S. A postpartum sleep and fatigue intervention feasibility pilot study. Behav Sleep Med 2018;16:185-201.

49 Moher D, Shamseer L, Clarke M, et al. Preferred reporting items for systematic review and meta-analysis protocols (PRISMA-P) 2015 statement. Syst Rev 2015;4:1.

50 Moher D, Liberati A, Tetzlaff J, et al. Preferred reporting items for systematic reviews and meta-analyses: the PRISMA statement. BMJ 2009;339:b2535.

51 Shamseer L, Moher D, Clarke M, et al. Preferred reporting items for systematic review and meta-analysis protocols (PRISMA-P) 2015: elaboration and explanation. BMJ 2015;350:g7647.

52 Moore GF, Audrey S, Barker M, et al. Process evaluation of complex interventions: medical Research Council guidance. BMJ 2015;350:h1258.

53 Grant A, Treweek S, Dreischulte T, et al. Process evaluations for cluster-randomised trials of complex interventions: a proposed framework for design and reporting. Trials 2013;14:15.

54 Baranowski T, Stables G. Process evaluations of the 5-a-day projects. Health Educ Behav 2000;27:157-66.

55 Higgins JPT, Altman DG, Gøtzsche PC, et al. The cochrane collaboration's tool for assessing risk of bias in randomised trials. BMJ 2011;343:d5928

56 Sterne JA, Hernán MA, Reeves BC, et al. ROBINS-I: a tool for assessing risk of bias in non-randomised studies of interventions. BMJ 2016;355:i4919.

57 Higgins JPT, Thompson SG. Quantifying heterogeneity in a metaanalysis. Stat Med 2002;21:1539-58.

58 Egger M, Davey Smith G, Schneider M, et al. Bias in meta-analysis detected by a simple, graphical test. BMJ 1997;315:629-34.

59 Balshem H, Helfand M, Schünemann HJ, et al. Grade guidelines: 3. rating the quality of evidence. J Clin Epidemiol 2011;64:401-6.

60 Guyatt GH, Oxman AD, Vist GE, et al. Grade: an emerging consensus on rating quality of evidence and strength of recommendations. BMJ 2008;336:924-6. 